\title{
The Design of Wire Feeding Mechanism for Desktop 3D Printer
}

\author{
Wang Haiyan ${ }^{1}$, Jiang Yunchun ${ }^{2}$, Song Aili ${ }^{3}$, Wang $\mathrm{Na}^{4}$ \\ Qingdao Huanghai University Qingdao China \\ E-mail: 541101645@qq.com
}

\begin{abstract}
D printing technology has developed for decades in our country, desktop 3D printers are gradually used popularly. Focused on the problems of large friction and instability of the wire feeder in the desktop 3D printer of FDM technology. The mechanisms of the internal and external wire feeding are studied and designed separately. The structure of crank slider and extensible beam are adopted by the external wire feeding mechanism, finite element analysis and optimization of design for frame, while increased the auxiliary trimming device for the internal wire feeding, thereby improving the performance of the wire feeding mechanism, reducing wire feed resistance, ensuring the stable of nozzle, improving printing quality.
\end{abstract}

Keywords: 3D Printing Technology; Wire Feeding Mechanism; Finite element analysis

\section{Introduction}

The 3D printer based on FDM technology is to extruded and pile up a solid body layer by layer according to the set path by heating ABS, PLA and other wire materials to be melted. Raw material in the initial solid state is extruded to a semi-liquid melt, wire feeding method has a great influence on the semi-liquid raw material's extrusion force. Wire resistance is the main reason affecting the instability of nozzle's wire. It solves the problem of the wire feeder's big friction, raw material supply instability, by changing the design of the internal and external wire feeding mechanisms.

\section{The design of external wire feeding mechanism}

The banknote withdrawal method in traditional banknote register mainly includes vacuum suction and friction withdrawal. Comprehensive comparison of the advantages and disadvantages of these two methods of banknote withdrawal, combined with the actual needs of the supermarket automatic change machine, choose the friction withdrawal which pays quickly, low cost, and technology perfect. This device is compact, occupies less space, widely used in ATM machines and bank tellers machines with a higher use proportion.

\section{The design scheme}

At present, desktop 3D printer wire disc mainly includes $0.5 \mathrm{~kg}, 1.0 \mathrm{~kg}$ and other specifications, According to the inconsistencies of the disk diameter size for different weight, design adjustable external wire feeding mechanism to adapt to different inside diameter. Reduce the wire friction resistance as much as possible, set spindle support structure, combine self-lubricating bearings to avoid wire broken, product defects caused by lack of wire material. According to the experimental data to analysis and select the design scheme of crank slider structure, shown in Figure 1. 


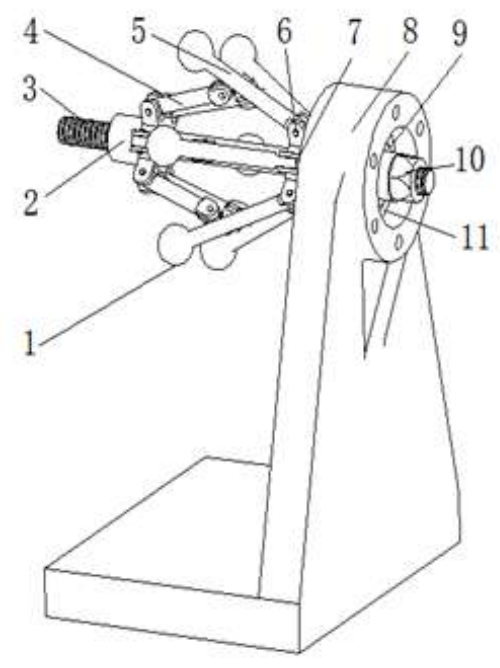

\begin{tabular}{|c|c|}
\hline 1 & fixed sleeve \\
\hline 2 & self-tightening right ring \\
\hline 3 & support shaft \\
\hline 4 & support diagonal rod \\
\hline 5 & support top rod \\
\hline 6 & pin \\
\hline 7 & self-tightening left ring \\
\hline 8 & support frame \\
\hline 9 & sleeve \\
\hline 10 & tighten nut \\
\hline 11 & bearing \\
\hline
\end{tabular}

Fig. 1 structure diagram

\section{The design of specific structure}

(1) The design of support shaft

The support shaft is one of the key structure of the external wire feeding mechanism, is the main force components. The material is ordinary carbon steel Q200, because it is not directly connected with the motor and the torque is small, its own weight should be considered in the design, according to the extrusion calculation.

Preliminary, the shaft diameter is $7 \mathrm{~mm}$, the squeezing pressure is $50 \mathrm{~N}$, the squeeze stress is by calculation. The allowable stress of material is $200 \times 10^{6} \mathrm{pa}>\sigma_{b s}$, meets the requirements, due to a key groove on the shaft, the calculated value is increased $3 \%$, take $8 \mathrm{~mm}$.

The high-speed rotation is not appeared in external wire feeding. The external wire feeding can withstand pure axial force, the deep groove ball bearing is chosen. In order to make the selected shaft diameter adapt to the bearing diameter, the 6022 of bearing type is selected, the relevant parameters is $d=10 \mathrm{~mm}, \mathrm{~B}=9 \mathrm{~mm}$. Diameter and length of the shaft section, according to the selected bearing, the diameter of combining with shaft is $10 \mathrm{~mm}$, bearing width is $B=9 \mathrm{~mm}$, in order to accurate location, the length of the bearing should be slightly shorter than $\mathrm{B}$, take $8 \mathrm{~mm}$.

Due to axial positioning of the bearing, the shoulder should be designed to meet the requirements. The total width of the bearing cover is $12 \mathrm{~mm}$, and at the same time, in order to facilitate handling and lubrication, the surface distance is $20 \mathrm{~mm}$.

(2) The design of support frame

The support structure is designed as extensible beam, which is simple and wide. According to the maximum outer diameter of the raw material disk is $200 \mathrm{~mm}$, the maximum width is $66 \mathrm{~mm}$, the specific dimensions of the design support frame, shown in Figure 2. 

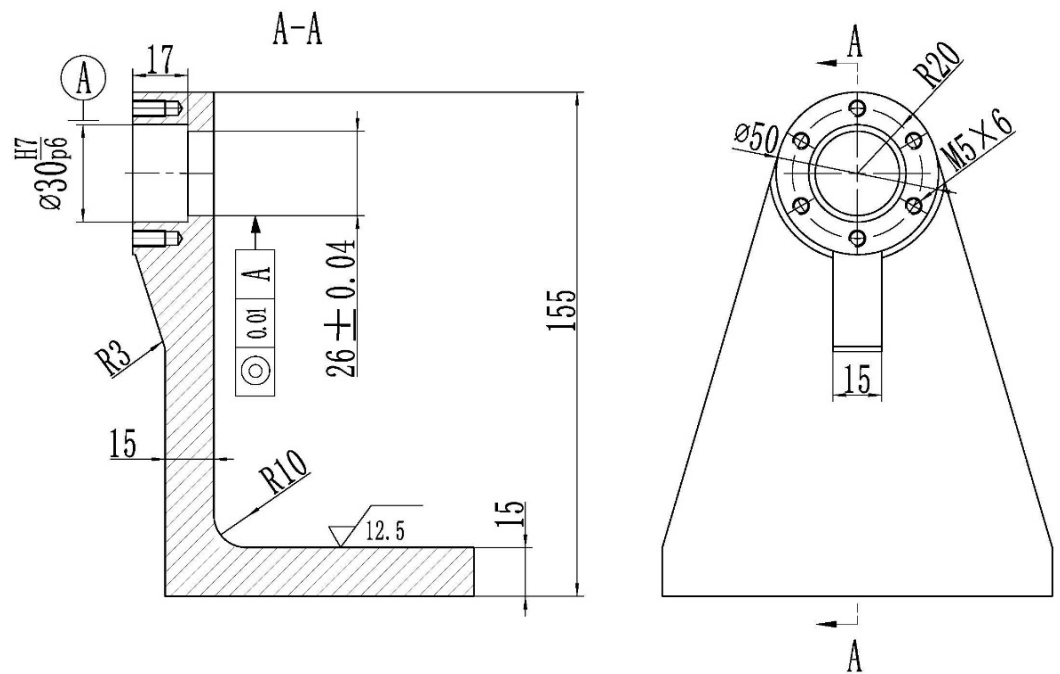

Fig. 2 Three views of the support frame

Due to coordinating the bearing, the width of the upper center hole is designed to be $17 \mathrm{~mm}$ and the diameter is $30 \mathrm{~mm}$. The bottom dimension is designed to be $95 \mathrm{~mm} \times 120 \mathrm{~mm} \times 15 \mathrm{~mm}$, the upper circle diameter is $50 \mathrm{~mm}$. The initial height of the support frame is designed to be $155 \mathrm{~mm}$ and the margin is large enough to be replaced by different specifications of wire disks. The adjustable diameter range is $22.28 \sim 83.88 \mathrm{~mm}$ and the maximum and minimum size is shown in Figure 3 and Figure

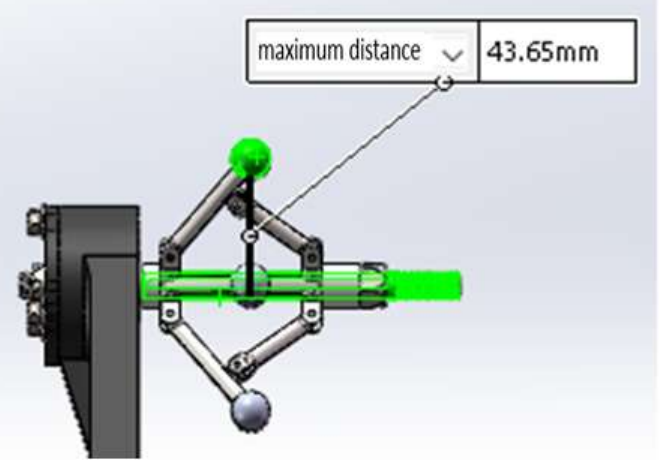

Fig. 3 the maximum size of support

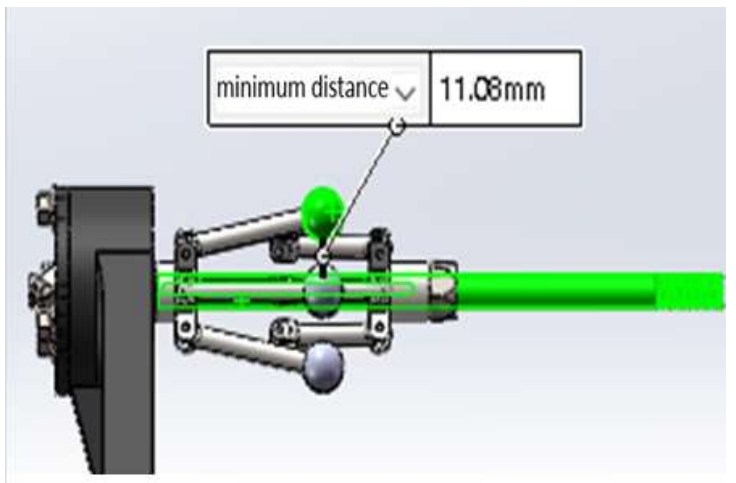

Fig. 4 the minimum size of support

(3) The analysis and optimization of support frame

During the analysis of the finite element, the imported 3D models are discretized so that they become finite tiny points, reducing the computational difficulty and simplifying the solution process. Analysis the discretization of the model is based on the mechanical properties, the component material needs to be defined in advance, so the gray iron is chosen as the material for the external wire support.

The quality of external support components is $1.5 \mathrm{~kg}$, although the quality of the raw materials currently used is $2 \mathrm{~kg}, 5 \mathrm{~kg}$ raw material is used in a few cases. The quality of material is set to be $6.5 \mathrm{~kg}$ initially. From the force point, the main vertical force of the component is $65 \mathrm{~N}$. The grid details are set firstly, all of them are gridded, the result is: The total segments is 649225 , the total units is 410048. Running iterative calculation, according to the analysis of the stress, strain and other cloud distribution, the overall grid can not meet the analysis requirements, and there are strange stress areas. 
The mesh parameters are further set and the mesh region is refined, the regions appeared stress singularity are subdivided. The refinement unit of mesh parameter is set as 2 , the ratio is 1.1 , the basic unit is 5 , and the mesh is generated by free transition. The radius of the fillet is changed to $5 \mathrm{~mm}$, a radius of $10 \mathrm{~mm}$ is added at the corner, and then iteration calculations are made. Run the results, the stress and displacement clouds are shown in Figure 5 and Figure 6.

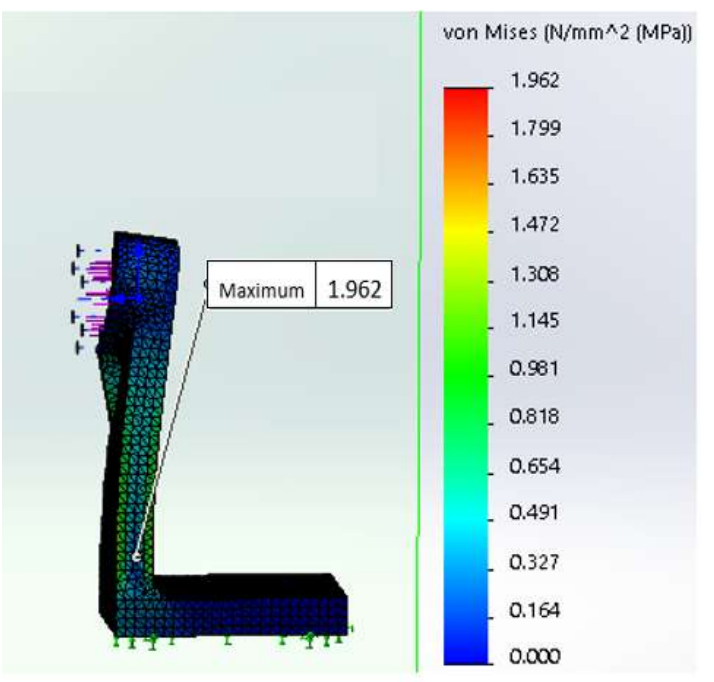

Fig. 5 stress cloud

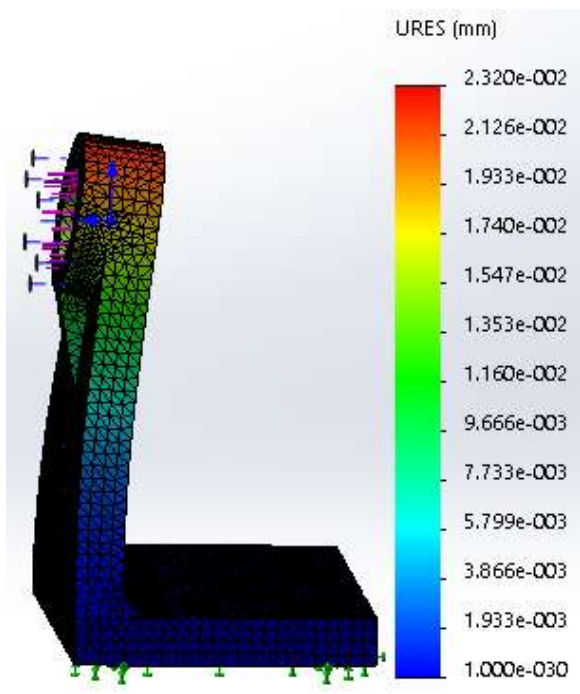

Fig. 6 displacement cloud

The maximum stress of the stress cloud is $1.962 \mathrm{Mpa}$, which is far less than the allowable stress of the material 200Mpa. The stress distribution of the components is discrete and there is no stress singularity accumulation. The maximum displacement of the cloud is $0.0232 \mathrm{~mm}$, does not belong to macro variables.

In summary, the support frame of external wire feeding can support different loads of raw materials during normal operation, and will not occur major deformation or stress destruction due to the weight of raw materials. The design dimensions and raw materials meet the requirements of normal use.

\section{The design of the internal wire feeding mechanism}

The internal wire feeding mechanism is the main structure of the heating and extruding device, a stepper motor is used to drive the wire feeding wheel, the solid raw material is pushed by the tooth of the wire feeding gear, and the wire is sent to the wire feeding tube through cooperation with the driven wheel, then into the heating device. Extrusion has three methods: piston, vane pump, plunger pump. Considering the device performance and design complexity, the piston extrusion device is used, the design has low precision control, small overall quality, low processing costs, short development cycle. Improved heating extrusion device is shown in Figure 7. 


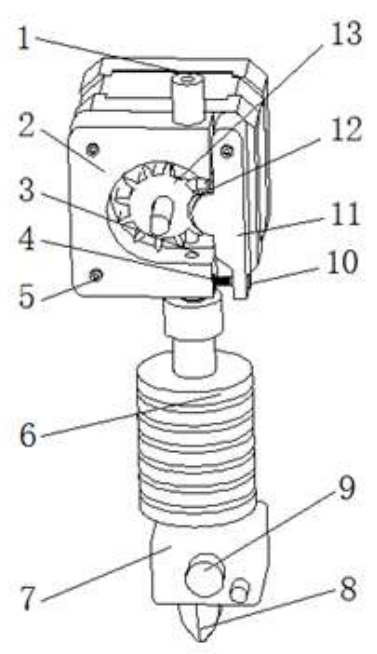

\begin{tabular}{|c|c|}
\hline 1 & wire feeding motor \\
\hline 2 & raw material model \\
\hline 3 & support shaft \\
\hline 4 & Adjustable spring \\
\hline 5 & set screw \\
\hline 6 & radiating pipe \\
\hline 7 & heating lead and thermocouple \\
\hline 8 & nozzle \\
\hline 9 & heating rod \\
\hline 10 & adjustable countersunk screw \\
\hline 11 & Fixed piece \\
\hline 12 & auxiliary wire wheel \\
\hline 13 & active wire wheel \\
\hline
\end{tabular}

Fig. 7 Heating extrusion device structure diagram

The auxiliary trimming function is added on the basis of the internal wire feeding mechanism of the existing extrusion heating device. The distance between auxiliary and active wire feeding gear and the raw material model is adjusted by the spring, to improve the active contact between the active wire feeding gear and the raw material model, and solve the problem of feeding inability or pulling off of the wire effectively.

The motor provides power for the extrusion heating device. According to the evaluation of the quality of the three-dimensional model, the bearing weight of $\mathrm{X}, \mathrm{Y}$ and $\mathrm{Z}$ axis does not exceed $2 \mathrm{~kg}$ and the bearing maximum of the $\mathrm{Z}$ axis is $2 \mathrm{~kg}$. The motor $2 \mathrm{HS} 4013 \mathrm{~A} 4$ is chosen, which is biphasic 42 stepper motor.

\section{Conclusion}

Based on fully understanding the weight of original raw materials and the size of wire disk, the structure of crank slider is adopted in the external wire feeding mechanism to realize self-tightening cooperation with different diameter wire disks, reduce the resistance of wire drawing and effectively avoid the problem of the discontinuous supply of raw materials. At the same time, the internal wire feeding structure has also been improved, the auxiliary wheel fine-tuning structure is set to improve the pressure between feed roller and wire and avoid pressure reduction caused by wire wheel wear.

\section{References}

[1] Wang Cancai.3D printing development status analysis [J]. Screen Printing, 2012 (9): 42-46.

[2] Niu Xi. The structural Analysis and Design of Desktop 3D Printers [J] .Design, 2015,04: 94-96.

[3] Liu Xinling.3D printer and its working principle [J]. Network and Information, 2012,2: 30. 